\title{
Starlike dendrimers in solutions: Structural properties and internal dynamics
}

\author{
Silke Rathgeber ${ }^{\mathrm{a})}$ \\ Max-Planck Institut für Polymerforschung, Polymer Physik, D-55128 Mainz, Germany \\ Michael Monkenbusch \\ Forschungszentrum Jülich GmbH, Institut für Festkörperforschung, D-52425 Jülich, Germany
}

James L. Hedrick and Mikael Trollsås

IBM Almaden Research Center, San Jose, California 95120-6099

Alice P. Gast

Department of Chemical Engineering, Masschusetts Institute of Technology, Cambridge, Massachusetts 02139

(Received 23 August 2006; accepted 26 September 2006; published online 28 November 2006)

\begin{abstract}
We measured the shape and the internal dynamics of starlike dendrimers under good solvent conditions with small-angle neutron scattering and neutron spin-echo (NSE) spectroscopy, respectively. Architectural parameters such as the spacer length and generation were varied in a systematic manner. Structural changes occurring in the dendrimers as a function of these parameters are discussed, i.e., in terms of the fractal dimension and deviations of the radius of gyration from the Gaussian value. A first cumulant evaluation of the NSE spectra for each scattering vector $q$ separately yields the length scale dependent relaxation rates. We observe a local minimum in the normalized relaxation rates $\Omega(q) / q^{3}$ on length scales corresponding to the overall dendrimer dimension. The dynamics is discussed within a Rouse-Zimm approach generalized to the case of starlike dendrimers of arbitrary geometry. The model allows an identification of the modes contributing to the relaxation of the dendrimer in the $q$ and time range of the NSE experiment. The local minimum is due to collective breathing motions of (parts of) the dendrons relative to each other. Shape fluctuations are not observed. () 2006 American Institute of Physics.
\end{abstract}

[DOI: $10.1063 / 1.2364895]$

\section{INTRODUCTION}

Dendrimers, first synthesized by Vögtle and coworkers, ${ }^{1}$ are highly branched macromolecules with a regular, treelike architecture. Starting from a multifunctional core multifunctional monomers are added in a stepwise synthesis resulting in controlled branching. ${ }^{2}$ Each synthesis step adds a new generation and leads to a multiplication of the number of monomers constituting the dendrimers. In so-called starburst dendrimers, only multifunctional units are added and branching occurs in single monomeric steps. In starlike dendrimers linear spacers are added between the branching units. The dendrimer topology is defined by architectural parameters, which include the generation number $g$, the core and branching unit functionalities, $f$ and $m$, respectively, and the number of monomers between the branching units $n$. For our particular system $f=6, m=2, n=5,10,15,20$, and $g=0,1,2$. A schematic representation of a starlike dendrimer is shown in Fig. 1.

Possible applications of dendrimers are directly related to the peculiarities of the dendrimer architecture: these include a large number of (functional) terminal units, possible voids in the dendrimer interior, regular centrosymmetric

\footnotetext{
${ }^{a)}$ Author to whom correspondence should be addressed. Electronic mail: s.rathgeber@mpip-mainz.mpg.de
}

branching, and a rather compact, globular shape, in particular, in the case of high generation dendrimers. Applications can be found in biology and medicine such as the use as host-guest systems for controlled drug release applications, in virus inhabitation, as gene transfer agents, or nuclear magnetic resonance (NMR) contrast agents, but they also can be used as catalysts for surface modification and manipulation or photochemical and optical applications such as light harvesting. For a comprehensive overview see Ref. 3 and references therein.

Their high application potential and the special features of the dendrimer architecture initiated a large number of experimental studies, ${ }^{4-11}$ computer simulations, ${ }^{10-23}$ as well as theoretical investigations ${ }^{12,16,24-28}$ addressing the structural properties of dendrimers. A more comprehensive overview is given in Refs. 10 and 12. Even though the above mentioned applications are not only determined by the dendrimer structure but also by their dynamical properties, much less attention has been drawn to the flexibility, i.e., internal dynamics of dendrimers. Experimental studies on the internal dynamics in solutions include predominantly NMR investigations $^{29-34}$ and some experiments using neutron spin-echo (NSE) spectroscopy. ${ }^{8,9}$ The latter are summarized in more detail below. There are also a few computer simulations ${ }^{16,17,35-37}$ and theoretical studies ${ }^{24,27,38-41}$ addressing this topic. 


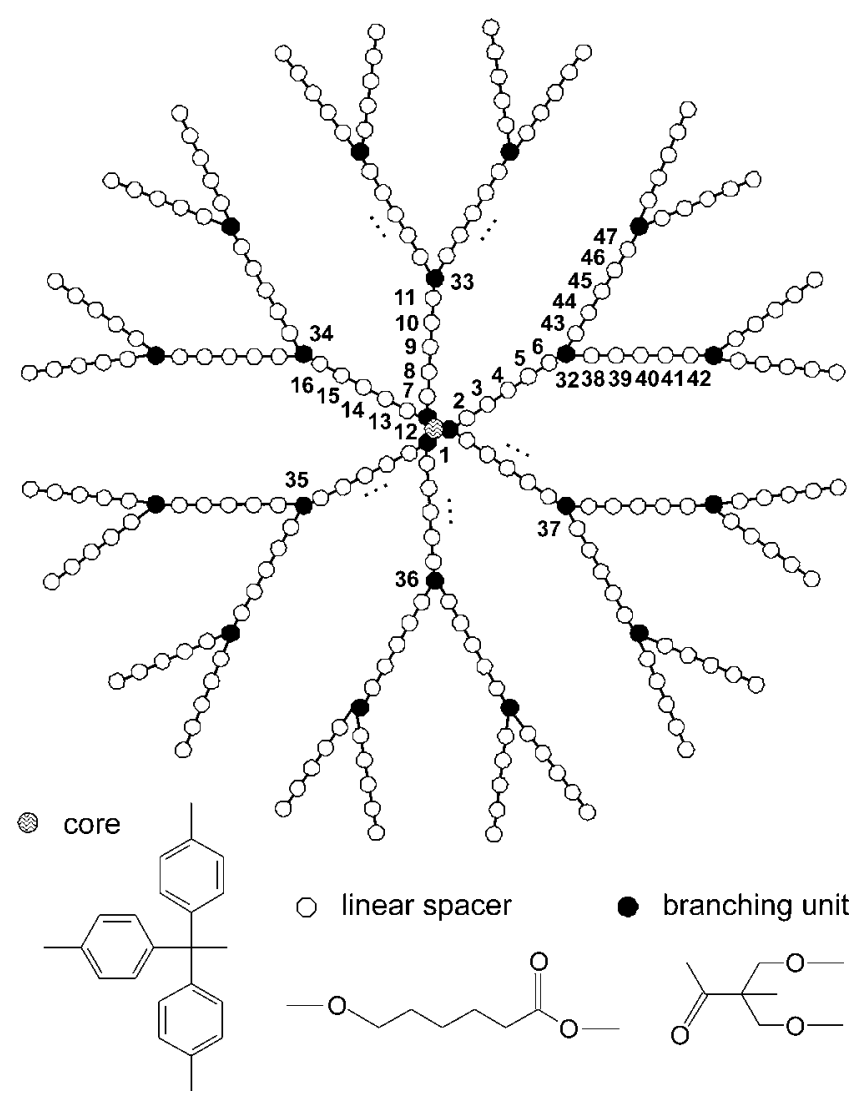

FIG. 1. Sketch of the $g 2 n 5 f 6$ starlike dendrimer.

Due to the high branching densities the segment densities in the dendrimer interior can become rather high, almost liquidlike for high generation dendrimers. Thus, on one side dendrimers will exhibit some sort of colloidal character, reflected, for example, in their well-defined compact shape, on the other side dendrimers are polymeric entities with a loose substructure which should allow some flexibility.

In previous measurements on starburst poly(amido amine) (PAMAM) dendrimers in dilute solutions with NSE spectroscopy, only center-of-mass (c.m.) diffusion of homogeneous spheres was detected. ${ }^{8}$ On the length scales covered by NSE spectroscopy the experiment should be sensitive to the internal dynamics which turns out to be too slow compared to the c.m. diffusion to be resolved. These findings are a consequence of the well-defined globular shape of the starburst dendrimers exhibiting very high polymer concentrations in the interior. Stark et al. ${ }^{9}$ conducted NSE experiments on carbosilane dendrimers with perfluorinated end groups. The authors observed an extrafast relaxation process leading to deviations from translational diffusive behavior and interpreted their results in terms of shape fluctuations, i.e., relaxation of ripples on the dendrimer surface. Due to the incompatibility between the end groups and the dendrimer interior, the end groups form a rather compact surface layer. The interpretation of the extrafast relaxation process in terms of form fluctuations implies not only a well-defined incompressible surface but also a low viscosity liquidlike interior and it cannot be excluded that it is due to internal density fluctuations rather than due to shape fluctuations. The experimental results on the dynamics of dendrimers reported so far reflect the compact shape that means the colloid-like character of dendrimers rather than their loose, polymeric nature. Previous studies by Richter and co-workers ${ }^{42-44}$ on highfunctionality star polymers, another class of highly branched, compact polymers, with NSE spectroscopy revealed a different dynamic behavior. On large length scales NSE spectroscopy resolves c.m. diffusion but on local length scales highfunctionality star polymers undergo internal Zimm dynamics. On intermediate length scales of the order of the dendrimer dimension the internal dynamics is slowed down compared to the Zimm dynamics due to breathing motions where the arms in the star polymer collectively are moving against each other. These dynamic peculiarities are reflected in the dependence of the relaxation rates $\Omega(q)$ on the scattering vector $q$. $\Omega(q)$ can be obtained by a first cumulant evaluation of the NSE relaxation spectra. The collective, breathing motions lead to a local minimum of $\Omega(q) / q^{3}$ at $q$ values corresponding to the length scales of the overall dimension of the star polymers. At high $q$ values $\Omega(q) / q^{3}$ enters a plateau as expected for internal Zimm relaxation on local length scales for which $\Omega(q) \propto q^{3}$ is expected. The upturn at small $q$ values is due to the characteristic $\Omega(q) \propto q^{2}$ dependence of free translational c.m. diffusion. In this paper we report our results on the dynamic behavior of poly ( $\varepsilon$-caprolactone) dendrimers in dilute solutions measured with NSE spectroscopy. The experiment is sensitive to the internal dynamics and we observe similar dynamic behavior as found by Richter and co-workers ${ }^{42-44}$ for highfunctionality star polymers. Shape fluctuations are not a relevant relaxation mechanism for the starlike dendrimers. We discuss differences between the dynamics of the starlike and starburst dendrimers in terms of the differences in the shape of both types of dendritic structures. Whereas starburst dendrimers are surface fractals with a rather compact shape, ${ }^{8}$ starlike dendrimers are mass fractals with a more extended density profile and lower polymer concentrations in the dendrimer interior. ${ }^{45}$ As in the case of the high-functionality star polymers, collective breathing motions of (parts of) the dendrons in the dendrimers lead to a slowing down of the internal dynamics on length scales corresponding to the dendrimer dimension. However, due to the fact that the dendrimer topology is more complex compared to the architecture of the star polymers, the spectrum of eigenmodes contributing to the structural relaxation is also more complex and involves more extensive theoretical considerations.

Theoretical studies on the internal dynamics of dendrimers include theories in the framework of the Rouse model ${ }^{24}$ as well as in the Rouse-Zimm model under consideration of hydrodynamic interactions, both with ${ }^{38-40}$ and without ${ }^{27,39,41}$ excluded volume interaction, respectively. We followed the formalism of the Rouse-Zimm model of Cai and Chen ${ }^{41}$ for the special case of starburst dendrimers with $f=3$ and $m=2$ and generalized the theory to the case of starlike dendrimers with arbitrary architecture, i.e., arbitrary $f, m$, and $n$. In this way we obtain information about the spectrum and the physical nature of the eigenmodes contributing to the structural relaxation of the dendrimers. In particular, we are able to 
assign which normal modes give a significant contribution to the relaxation of the dynamic structure factor on length and time scales of the NSE experiment.

In summary, the focus of our paper is to obtain a descriptive insight into the relaxation mechanism of dendrimers and how their internal dynamics is related to their structural properties.

\section{THEORY}

\section{A. Rouse-Zimm dynamics}

Assuming Gaussian distribution for the time dependent interbead distances the intermediate structure factor for dilute solutions of identical scatterers can be written in terms of the coordinates of the scattering units $\boldsymbol{r}_{i}$ as

$$
S(q, t)=N_{t}^{-2} \sum_{i, j=1}^{N_{t}} \exp \left(-\frac{q^{2}}{6}\left\langle\left|\boldsymbol{r}_{i}(t)-\boldsymbol{r}_{j}(0)\right|^{2}\right\rangle\right),
$$

where $q$ denotes the norm of the scattering vector and $t$ the time. As sketched in Fig. 1 the macromolecule is described as an assembly of $N_{t}$ interconnected beads numbered from 1 to $N_{t}$ having identical translational friction coefficients. The beads are connected by $N_{t}-1$ bonds of mean square length $l^{2}$. The topology of the starlike dendrimers is determined by the architectural parameters, which include the functionalities of the core and branching units, $f$ and $m$, respectively, the generation number $g$, and the number of segments $n$ between the branching units. To obtain the evolution of the bead coordinates $\boldsymbol{r}_{i}$ with time $t$ the equation of motions for this coupled $N_{t}$ particle system has to be formulated. In the Rouse-Zimm approximation the Langevin equation reads

$$
\frac{\partial}{\partial t} \boldsymbol{r}_{i}(t)+\frac{\sigma}{\zeta} \sum_{j=1}^{N_{t}}[\boldsymbol{H} \boldsymbol{A}]_{i j} \boldsymbol{r}_{j}(t)=\boldsymbol{H} \boldsymbol{f}_{i}(t)
$$

where $\zeta$ is the monomeric friction coefficient. The surrounding, described as heat bath, acts on the test chain solely via the stochastic forces $f_{i}(t)$. Intramolecular interactions between neighboring monomers (beads) due to restoring forces originating from the entropy elasticity of polymers are taken into account by a harmonic potential of strength $\sigma=3 k_{B} T l^{-2}$, where $k_{B}$ denotes the Boltzmann constant and $T$ is the temperature. The symmetric force-constant matrix $\boldsymbol{A}$ of order $N_{t}$ takes into account the topological connectivity of the chain and the equilibrium bond-bond correlations. The construction of the connectivity matrix follows the procedure described in Ref. 41 and is described in the Appendix for starlike dendrimers with arbitrary architectrure.

The mobility matrix $\boldsymbol{H}$ is given by

$$
\boldsymbol{H}_{i j}=k_{B} T\left[\left(1-\delta_{i j}\right) \boldsymbol{T}_{i j}+\frac{\delta_{i j}}{\zeta} \underline{\underline{ }}\right] \text {, }
$$

where the so-called Oseen tensor,

$$
\boldsymbol{T}_{i j}=\frac{1}{8 \pi \eta\left|\boldsymbol{r}_{i j}\right|}\left(\underline{\underline{1}}+\frac{\boldsymbol{r}_{i j} \otimes \boldsymbol{r}_{i j}}{\boldsymbol{r}_{i j}^{2}}\right) \quad \text { with } i \neq j,
$$

accounts for nonlocalized interactions between monomers (beads) arising from long-ranged hydrodynamic interactions due to the flow field created in the fluid by the movements of all beads. Here, $\boldsymbol{\eta}$ is the solvent viscosity, $\boldsymbol{r}_{i j}=\boldsymbol{r}_{i}-\boldsymbol{r}_{j}$ and $\otimes$ denotes the outer product. In the Rouse-Zimm model, $\boldsymbol{H}$ (matrix of order $N_{t}$ ) is replaced by its average using the equilibrium conformational distribution function yielding

$$
\left\langle\boldsymbol{H}_{i j}\right\rangle=k_{B} T\left[\frac{\delta_{i j}}{\zeta}+\frac{\zeta_{r}}{\zeta} l\left\langle r_{i j}^{-1}\right\rangle\left(1-\delta_{i j}\right)\right] .
$$

The reduced friction coefficient,

$$
\zeta_{r}=\frac{\zeta}{6 \pi \eta l}
$$

is a measure of the hydrodynamic interactions representing the effective bead hydrodynamic radius in units of the bond length. Assuming Gaussian equilibrium distribution for the mean square interbead distances $\left\langle r_{i j}^{2}\right\rangle=\left\langle\left|\boldsymbol{r}_{i}-\boldsymbol{r}_{j}\right|^{2}\right\rangle,\left\langle r_{i j}^{-1}\right\rangle$ can be written as

$$
\left\langle r_{i j}^{-1}\right\rangle=\left\langle\left|\boldsymbol{r}_{i}-\boldsymbol{r}_{j}\right|^{-1}\right\rangle=\sqrt{\frac{6}{\pi\left\langle r_{i j}^{2}\right\rangle}} .
$$

Decoupling of the equations of motion, given in Eq. (2), is achieved by transforming the Cartesian bead coordinates $\boldsymbol{r}_{i}$ to normal coordinates $\boldsymbol{x}_{\alpha}$,

$$
\boldsymbol{r}_{i}=\sum_{\alpha=1}^{N_{t}} \boldsymbol{Q}_{i \alpha} \mathbf{x}_{\alpha},
$$

using the matrix $\boldsymbol{Q}$ of eigenvectors $\boldsymbol{Q}_{i \alpha}$ of the matrix $[\boldsymbol{H A}]$ which can be obtained by solving the eigenvalue problem

$$
[\boldsymbol{H} \cdot \boldsymbol{A}] \cdot \boldsymbol{Q}=\boldsymbol{Q} \cdot \Lambda \quad \text { with } \Lambda=\left\{\delta_{i \alpha} \lambda_{\alpha}\right\}
$$

The matrix of eigenvalues $\Lambda$ contains $N_{t}$ eigenvalues $\lambda_{\alpha}$ comprising an eigenvalue equal to zero corresponding to the c.m. diffusion and $N_{t}-1$ eigenvalues related to normal modes describing internal relaxations with relaxation rates $\tau_{\alpha}$ $=\left(\lambda_{\alpha} \sigma / \zeta\right)^{-1}$. The decoupled transformed equation of motion is

$$
\frac{\partial}{\partial t} \mathbf{x}_{\alpha}(t)+\tau_{\alpha}^{-1} \mathbf{x}_{\alpha}(t)=\boldsymbol{f}_{\alpha}^{*}
$$

Since the forces $f_{\alpha}^{*}$ are uncorrelated, the time correlation functions of the normal coordinates can be written as

$$
\begin{aligned}
& \left\langle\boldsymbol{x}_{\alpha}(t) \boldsymbol{x}_{\beta}(0)\right\rangle=\delta_{\alpha \beta}\left\langle\boldsymbol{x}_{\alpha}^{2}(0)\right\rangle \exp \left(-t \tau_{\alpha}^{-1}\right), \\
& \left\langle\left[\boldsymbol{x}_{0}(t)-\boldsymbol{x}_{0}(0)\right]^{2}\right\rangle=v_{0} 6 \frac{k_{B} T}{\zeta N_{t}} t,
\end{aligned}
$$

where $\left\langle\boldsymbol{x}_{\alpha}^{2}(0)\right\rangle=l^{2} \mu_{\alpha}^{-1}$. The elements $\mu_{\alpha}$ and $v_{\alpha}$ of the diagonal matrices $\boldsymbol{M}=\left\{\delta_{\alpha \beta} \mu_{\alpha}\right\}$ and $\boldsymbol{N}=\left\{\delta_{\alpha \beta} v_{\alpha}\right\}$ are the solutions of the equations

$$
\boldsymbol{M}=\boldsymbol{Q}^{T} \cdot \boldsymbol{A} \cdot \boldsymbol{Q} \text { and } \boldsymbol{N}=\boldsymbol{Q}^{-1} \cdot \boldsymbol{H} \cdot \boldsymbol{Q}^{-1 T},
$$

respectively. Transformation into Cartesian coordinates with Eq. (8) yields 


$$
\begin{aligned}
\left\langle\left|\boldsymbol{r}_{i}(t)-\boldsymbol{r}_{j}(0)\right|^{2}\right\rangle= & l^{2} \sum_{\alpha=2}^{N_{t}}\left(\boldsymbol{Q}_{i \alpha}^{2}+\boldsymbol{Q}_{j \alpha}^{2}\right) \mu_{\alpha}^{-1} \\
& -2 l^{2} \sum_{\alpha=2}^{N_{t}} \boldsymbol{Q}_{i \alpha} \boldsymbol{Q}_{j \alpha} \mu_{\alpha}^{-1} \exp \left(-t \tau_{\alpha}^{-1}\right) \\
= & \left\langle r_{i j}^{2}\right\rangle+2 l^{2} \sum_{\alpha=2}^{N_{t}} \boldsymbol{Q}_{i \alpha} \boldsymbol{Q}_{j \alpha} \mu_{\alpha}^{-1}\left[1-\exp \left(-t \tau_{\alpha}^{-1}\right)\right]
\end{aligned}
$$

where the mean square interbead distances can also be expressed in terms of the eigenvalues $\lambda_{\alpha}^{0}$ and eigenvectors $\boldsymbol{Q}_{\alpha}^{0}$ of $\boldsymbol{A}$, i.e., in the free-draining limit,

$$
\begin{aligned}
\left\langle r_{i j}^{2}\right\rangle=n_{i j} l^{2} & =l^{2} \sum_{\alpha=2}^{N_{t}}\left(\boldsymbol{Q}_{i \alpha}-\boldsymbol{Q}_{j \alpha}\right)^{2} \mu_{\alpha}^{-1} \\
& =l^{2} \sum_{\alpha=2}^{N_{t}}\left(\boldsymbol{Q}_{i \alpha}^{0}-\boldsymbol{Q}_{j \alpha}^{0}\right)^{2}\left(\lambda_{\alpha}^{0}\right)^{-1} .
\end{aligned}
$$

Here, $n_{i j}$ is the number of segments in the shortest path between two segments $i$ and $j$. Insertion of Eqs. (13) and (14) into Eq. (1) yields the final result for the intermediate structure factor $S(q, t)$. For $t=0$,

$$
P(q)=N_{t}^{-2} \sum_{i, j=1}^{N_{t}} \exp \left(-\frac{q^{2}}{6}\left\langle r_{i j}^{2}\right\rangle\right)
$$

the form factor $P(q)$ is obtained. The first cumulant, determined from the initial logarithmic decay of the intermediate scattering function,

$$
\Omega(q)=-\frac{d}{d t} \ln [S(q, t)]_{t=0},
$$

can be expressed in terms of the mobility matrix as follows:

$$
\Omega(q)=\frac{\sum_{i=1}^{N_{t}} \sum_{j=1}^{N_{t}}\left\langle\left(\boldsymbol{q} \boldsymbol{H}_{i j} \boldsymbol{q}\right) \exp \left(i \boldsymbol{q} \boldsymbol{r}_{i j}\right)\right\rangle}{\sum_{i=1}^{N_{t}} \sum_{j=1}^{N_{t}}\left\langle\exp \left(i \boldsymbol{q} \boldsymbol{r}_{i j}\right)\right\rangle} .
$$

Taking the average, first over all orientations and subsequently over all scalar interbead distances $r_{i j}$ with a Gaussian distance distribution, yields

$$
\begin{aligned}
\Omega(q)= & \frac{q^{2}}{P(q)} \frac{k_{B} T}{\zeta}\left[1+\frac{\zeta}{8 \pi \eta l N_{t}^{2}}\right. \\
& \left.\times \sum_{i=1}^{N_{t}} \sum_{j=1}^{N_{t}}\left(1-\delta_{i j}\right) l\left\langle r_{i j}^{-1}\right\rangle y\left(u_{i j}\right)\right],
\end{aligned}
$$

with $u_{i j}=q \sqrt{\left\langle r_{i j}^{2}\right\rangle / 6}$ and

$$
y\left(u_{i j}\right)=\left\{\begin{array}{l}
\frac{4}{3} \exp \left(-u_{i j}^{2}\right) \quad \text { with preaveraging } \\
\left(u_{i j}^{-3}+2 u_{i j}^{-1}\right) \exp \left(-u_{i j}^{2}\right) \int_{0}^{u_{i j}} \exp \left(t^{2}\right) d t-u_{i j}^{-2} \\
\text { without preaveraging, }
\end{array}\right.
$$

where for the calculation either the preaveraged [as given by Eq. (5) and used in the Rouse-Zimm model] or nonpreaveraged version of the mobility matrix given by Eqs. (3) and (4), respectively has been used. For good solvent conditions, $\left\langle r_{i j}^{2}\right\rangle$ given in Eq. (14) has to be replaced by $\left\langle r_{i j}^{2}\right\rangle=n_{i j}^{2 \nu} l^{2}$ where the Flory exponent $v$ equals $3 / 5$.

Once the segment length $l$ is determined from a fit of the static scattering spectra using Eq. (15), the monomeric friction coefficient $\zeta$ or the reduced friction coefficient $\zeta_{r}$ [see Eq. (6)] as a measure of the strength of the hydrodynamic interactions, respectively, is the only adjustable parameter in the determination of the first cumulant or the intermediate structure factor.

To emphasize the contribution of modes belonging to the same eigenvalue to the relaxation of the intermediate structure factor in a certain $q$ range, the quantity

$$
\begin{aligned}
R_{\alpha, \alpha+\varepsilon}(q)= & N_{t}^{-2} P(q)^{-1} \sum_{i, j=1}^{N_{t}} \exp \left[-\frac{q^{2}}{6}\left(\left\langle r_{i j}^{2}\right\rangle+2 l^{2}\right.\right. \\
& \left.\left.\times \sum_{\alpha}^{\alpha+\varepsilon} \boldsymbol{Q}_{i \alpha} \boldsymbol{Q}_{j \alpha} \mu_{\alpha}^{-1}\right)\right]
\end{aligned}
$$

can be plotted as a function of the scattering vector. $R_{\alpha, \alpha+\varepsilon}(q)$ gives the maximal contribution of all modes belonging to the same eigenvalue $\lambda_{\alpha}$ with degeneracy $\varepsilon$ to the decay of $S(q, t)$, i.e., in the limit of long times $(t \rightarrow \infty)$ in case all other modes are frozen in $t=0$.

\section{EXPERIMENTS}

\section{A. Samples}

The starlike dendrimers, based on aliphatic polyesters, were synthesized by a divergent growth approach. A hexahydroxyl-functional 2.2-bis(hydroxylmethyl)propionic acid (bis-MPA) derivate was used as initiator for the stannous-2-ethylhexanoate catalyzed living ring opening polymerization of linear poly( $\varepsilon$-caprolactone). Higher generations were obtained by functionalization of the so-obtained macroinitiators with protected bis-MPA, and subsequent deprotection and growth of linear poly( $\varepsilon$-caprolactone). More details of the synthesis of the dendrimers are discussed in Ref. 46. In this way starlike dendrimers were obtained with core and branching functionalities being $f=6$ and $m=2$, respectively.

The generation number was varied between $g=0$ and $g$ $=2$, where $g=0$ denotes the six arm star polymer. The lengths of the linear poly $(\varepsilon$-caprolactone) spacers between the branching units were $n=5,10,15$, and 20, respectively. Throughout the paper we will use $g$ " $X " n " X " f$ " $X$ " as 
sample identifier, where the generation, spacer length, and core functionality are always given in this order. A sketch of the structure is given in Fig. 1.

\section{B. Small-angle neutron scattering}

The small angle neutron scattering (SANS) experiments were performed on the NG3 30 m SANS instrument at the NIST Center for Neutron Research of the National Institute of Standards and Technology (NIST), Gaithersburg, MD. At a wavelength of $\lambda_{0}=0.6 \mathrm{~nm}$ with a bandwidth of $\Delta \lambda / \lambda_{0}$ $=0.15$ the covered range of scattering vectors was $0.55 \mathrm{~nm}^{-1} \leqslant q \leqslant 2 \mathrm{~nm}^{-1}$ using two detector distances $d=2$ and $10 \mathrm{~m}$. The source to sample aperture distance was set to $11.6 \mathrm{~m}$. As sample containers we used sealed, round Hellma quartz cuvettes with a path length of $2 \mathrm{~mm}$. All experiments were performed at a temperature of $T=(20 \pm 0.1){ }^{\circ} \mathrm{C}$ using a circulating fluid bath thermostat. The two-dimensional data were corrected via standard procedures for absorption, detector sensitivity, and background stemming from the deuterated toluene and empty cuvette and are converted to absolute intensities using a secondary standard. In a subsequent step the corrected data were radial averaged. Experimental $q$ resolution effects,

$$
\Delta q_{\mathrm{FWHM}}=\left[\left(q \Delta \lambda / \lambda_{0}\right)^{2}+\left(\Delta \theta 2 \pi / \lambda_{0}\right)^{2}\right]^{1 / 2},
$$

with an angular resolution of $\Delta \theta=4.322 \times 10^{-3} \mathrm{rad}$, were considered in the fitting routines for data evaluation using the formula suggested by Pedersen ${ }^{47}$ for the experimental $q$ resolution of a SANS instrument. Samples with concentrations between $1 \%$ and $0.25 \%$ in deuterated toluene have been measured. For $q$ values lower than $0.1 \mathrm{~nm}^{-1}$, the small influence of the structure factor due to interdendrimer interactions was corrected by Zimm extrapolation to zero concentration. To ensure an as best as possible background correction high$q$ data shown are taken from data sets obtained for samples with polymer concentrations of $1 \%$.

\section{Neutron spin-echo experiments}

The meutron spin-echo (NSE) experiments were carried out at the NSE spectrometer at the DIDO reactor of the Forschungszentrum Jülich $\mathrm{GmbH}$, Jülich, Germany. With five settings $\left(q=0.5,0.8,1.0,1.4 \mathrm{~nm}^{-1}\right)$ of the area detector a $q$ range from 0.26 up to $1.8 \mathrm{~nm}^{-1}$ was covered. The spectrometer was operated at an incident neutron wavelength of $0.8 \mathrm{~nm}$ with a bandwidth of $\Delta \lambda / \lambda_{0}=0.10$. With an angular resolution of $\Delta \theta=9.3 \times 10^{-3} \mathrm{rad}$, the $q$ resolution calculated from Eq. (21) varied between 0.1 and $0.2 \mathrm{~nm}^{-1}$ with $q$. With 14 Fourier time settings a time range between 0.1 and $22 \mathrm{~ns}$ was covered. As sample containers we used customized, sealed, rectangular Hellma quartz cuvettes with a path length of $4 \mathrm{~mm}$. The reduction of the multidetector data to $S(q, t) / S(q, 0)$ was done following the procedures described in Ref. 48. Experimental resolution was measured using Carbopack B purchased from Supelco, Inc. as fully elastic scatterer. For background correction, the deuterated solvent was measured over the full time range and subtracted from the sample signal with proper weighting factors. To obtain sufficient signal, measurements were performed on deuterated

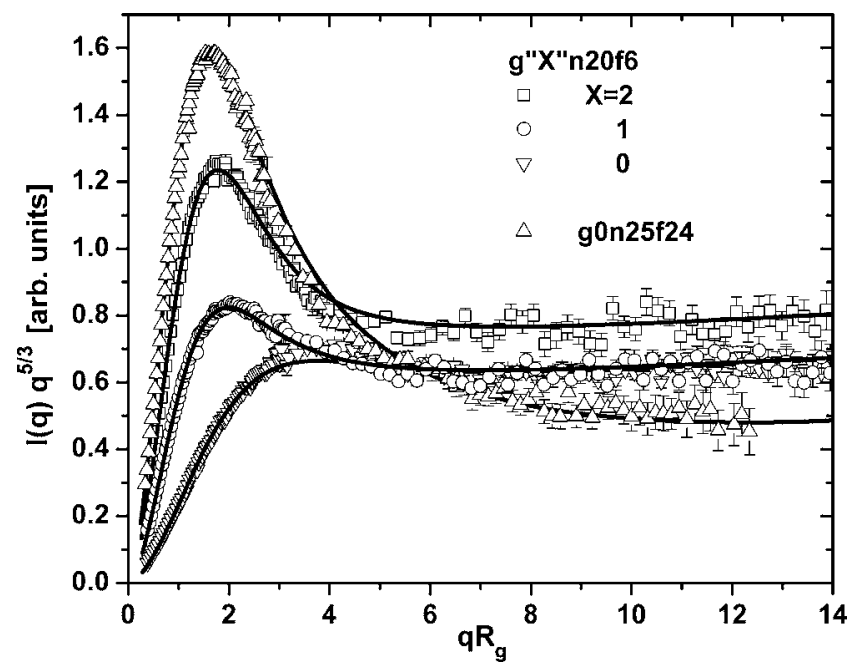

FIG. 2. SANS spectra of starlike dendrimers having different generation numbers $g=0,1$, and 2 but same spacer monomer number of $n=20$. The spectrum obtained for a high-functionality star polymer $g 0 n 25 f 24$ is also included. The solid lines show the results of a fit using Eq. (15).

toluene solutions containing 5 wt $\%$ polymer. Corrections effects arising from interdendrimer interactions are performed as described in Ref. 8.

\section{RESULTS AND DISCUSSION}

In Figs. 2 and 3 the form factors obtained for the starlike dendrimers in dilute toluene solutions are shown in a Kratky presentation generalized for good solvent conditions. Figure 2 presents the spectra as a function of the dendrimer generation $g=0-2$ for fixed spacer length $n=20$ and in Fig. 3 the spectra for different spacer lengths $n=5,10,15$, and 20 but fixed generation $g=2$ are shown. The scattering spectrum obtained for a high-functionality star polymer $g 0 n 25 f 24$ is also included in Fig. 2. The peak of the SANS spectra in the Kratky presentation becomes more pronounced with increasing dendrimer generation as well as with increasing spacer length. A more pronounced peak in the Kratky presentation

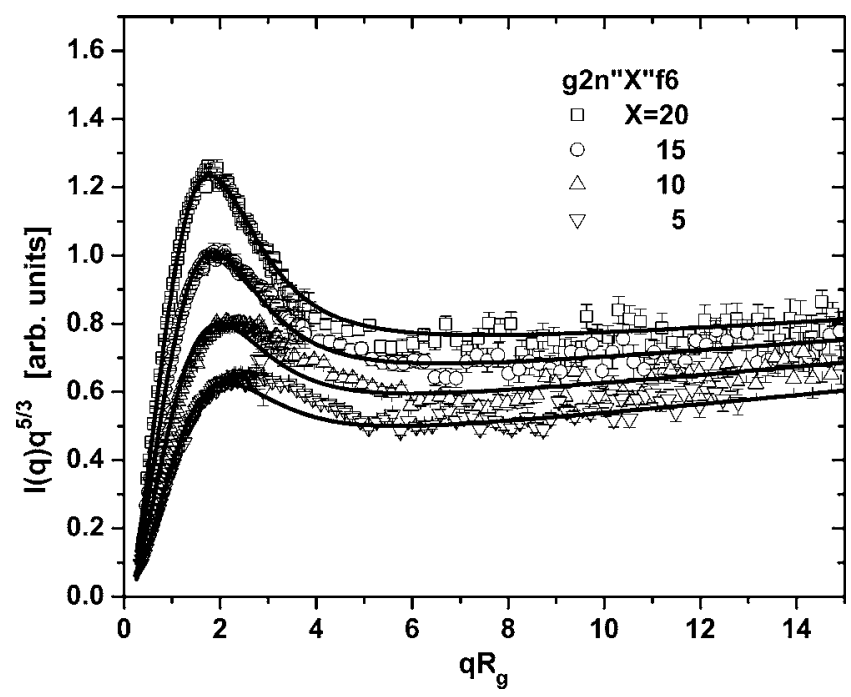

FIG. 3. SANS spectra of the starlike $g=2$ dendrimers having different spacer monomer numbers $n=5,10,15$, and 20. The solid lines show the results of a fit using Eq. (15). 
TABLE I. Structural properties of the starlike dendrimers and of the high-functionality star polymer and homopolymer.

\begin{tabular}{|c|c|c|c|c|c|c|c|}
\hline Sample & $g$ & $n$ & $f$ & $\begin{array}{c}R_{g} \\
(\mathrm{~nm})\end{array}$ & $\begin{array}{c}R_{g}^{\text {Gauss }} \\
(\mathrm{nm})\end{array}$ & $\begin{array}{c}\Delta \\
(\%)\end{array}$ & $D_{f}$ \\
\hline \multicolumn{8}{|c|}{ Variation of spacer length } \\
\hline$g 2 n 5 f 6$ & 2 & 5 & 6 & $6.39 \pm 0.07$ & 3.98 & 51 & $1.91 \pm 0.01$ \\
\hline$g 2 n 10 f 6$ & 2 & 10 & 6 & $7.75 \pm 0.09$ & 5.30 & 46 & $2.10 \pm 0.02$ \\
\hline$g 2 n 15 f 6$ & 2 & 15 & 6 & $8.40 \pm 0.08$ & 6.32 & 33 & $2.35 \pm 0.02$ \\
\hline$g 2 n 20 f 6$ & 2 & 20 & 6 & $8.69 \pm 0.06$ & 7.18 & 21 & $2.52 \pm 0.01$ \\
\hline \multicolumn{8}{|c|}{ Variation of generation } \\
\hline$g 1 n 20 f 6$ & 1 & 20 & 6 & $6.97 \pm 0.04$ & 5.72 & 22 & $2.15 \pm 0.02$ \\
\hline$g 0 n 20 f 6$ & 0 & 20 & 6 & $4.94 \pm 0.08$ & 4.00 & 23 & $1.64 \pm 0.01$ \\
\hline \multicolumn{8}{|c|}{ Reference samples } \\
\hline$g 0 n 25 f 24$ & 0 & 25 & 24 & $6.17 \pm 0.07$ & 3.83 & 61 & $2.78 \pm 0.02$ \\
\hline$g 0 n 144 f 1$ & 0 & 144 & 1 & $5.39 \pm 0.06$ & 5.39 & 0 & $1.68 \pm 0.02$ \\
\hline
\end{tabular}

indicates a more compact overall shape. Thus, we can conclude that the overall shape of the starlike dendrimers becomes more compact with increasing spacer length and increasing generation but always stays less compact than that of a high-functionality star polymer. The solid lines in Figs. 2 and 3 show the results of a simultaneous fit of all spectra using Eq. (15) with $\left\langle r_{i j}^{2}\right\rangle=n_{i j}^{2 \nu} l^{2}$ for good solvent conditions. The only adjustable parameter of the fit is the segment length $l$ which was determined to be $l=0.75 \pm 0.03 \mathrm{~nm}$ for the starlike dendrimers and $l=1.13 \pm 0.01 \mathrm{~nm}$ for the highfunctionality star polymer. In a single fit of the spectra the segment length $l$ varied only unsystematically within the error bars, thus, the segment length was set equal for all starlike dendrimers. We obtain rather good agreement between theory and experiment for the dendrimers having long spacers (series $g$ " $X " n 20 f 6$ ) and the high-functionality star polymer. For the $g 2 n " X " f 6$ series the agreement worsens with decreasing spacer length. This observation is consistent with the results obtained using the Beaucage model which was discussed in a previous publication. ${ }^{45}$ The Beaucage model parametrizes the form factor of a fractal object into two contributions. ${ }^{49}$ The first contribution, a Guinier function, describes the scattering at low $q$ values and includes the radius of gyration $R_{g}$ of the scattering object as an adjustable parameter. The second contribution, a power-law decay at high $q$ values, describes the scattering originating from the internal fractal structure of the particle. The exponent of this power-law decay is inversely proportional to the fractality $D_{f}$ of the substructure. The results for $R_{g}$ and $D_{f}$ are summarized in Table I for all samples and are presented in Figs. 3 and 4 of Ref. 45. As references, values are also given for the highfunctionality star polymer $g 0 n 25 f 24$ and a homopolymer $g 0 n 144 f 1$. The radius of gyration of a Gaussian dendrimer $R_{g}^{\text {gauss }}$ (as in Table I) were calculated using Eq. (5) given in Ref. 45 and are included in Table I together with the deviations from Gaussian behavior in terms of $\Delta=R_{g} / R_{g}^{\text {gauss }}-1 . \Delta$ significantly increases with decreasing spacer length but is independent of the generation for the dendrimers with long spacers. In conclusion, an increasing generation does not cause additional stretching of the dendrons. The gain in volume obviously compensates the increasing sterical crowding in the outer shells. Reducing the spacer length reduces the accessible volume with the result that the dendrons stretch out for compensation. The fractality increases from the theoretical value expected for a free, linear chain under good solvent conditions with increasing spacer length and generation but always stays smaller than that obtained for the highfunctionality star polymer. The fact that shorter spacers lead to lower fractal dimensions can also be taken as indication that the dendrons are stretched and a space filling arrangement is prevented. Hence, the deviations in Fig. 3 between the theoretical curves and the experimental spectra obtained for the dendrimers with short spacers $(n=5$ and 10) are most likely due to discrepancies from Gaussian statistics which is the presumption for the derivation of Eq. (15).

In Fig. 4 the neutron spin-echo spectra obtained for a $g 2 n 10 f 6$ dendrimer are shown covering a $q$ range from $q$ $=0.256$ up to $1.300 \mathrm{~nm}^{-1}$. The dashed lines show a fit to the relation

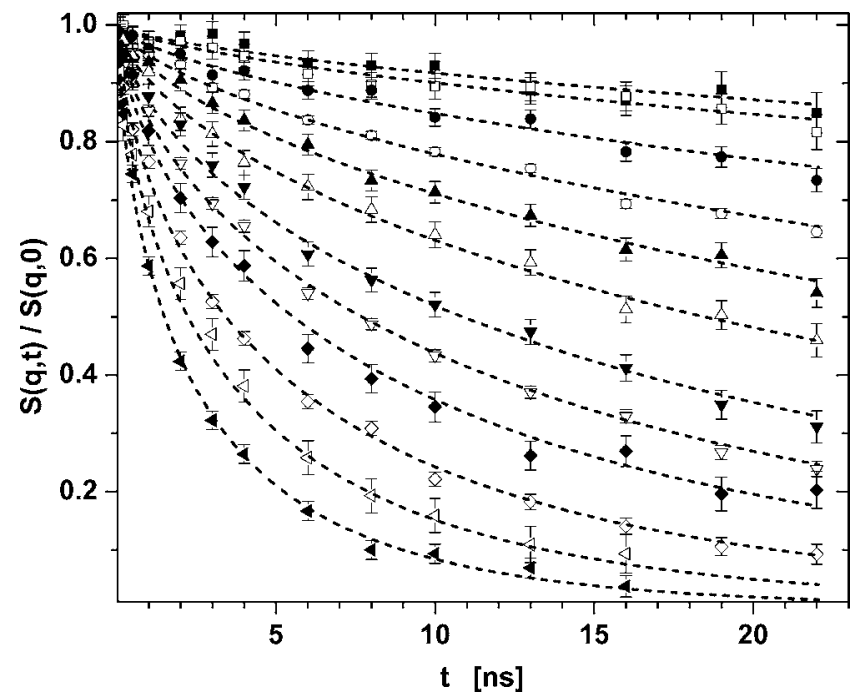

FIG. 4. NSE spectra obtained for the $g 2 n 10 f 6$ dendrimer for different $q$ values. From top to bottom: $q=(0.256,0.326,0.410,0.500$, $0.562,0.636,0.706,0.800,0.856,1.000,1.112,1.300) \mathrm{nm}^{-1}$. Dashed lines show the results of a fit using Eq. (22). 


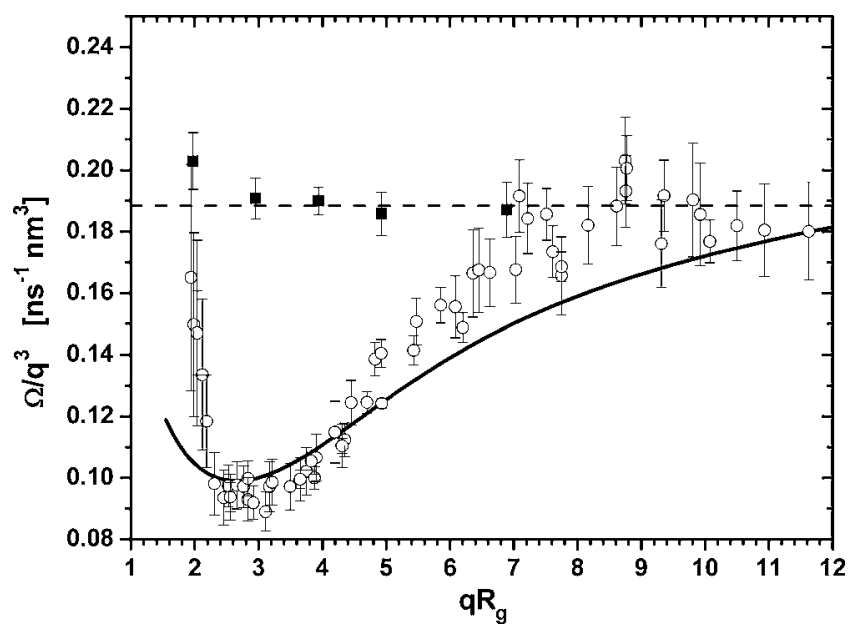

FIG. 5. Results from a first cumulant evaluation of the NSE spectra obtained for the $g 2 n 10 f 6$ dendrimer (open symbols) and a $g 0 n 144 f 1$ homopolymer (filled symbols). The first cumulant is normalized to the $q^{3}$ dependence expected for internal Zimm dynamics. The solid line shows a result of a fit using Eqs. (18) and (19) without preaveraging. The dashed line marks the average value for $\Omega(q) / q^{3}$ obtained for the homopolymer omitting the value for the smallest $q$ value.

$$
\frac{S(q, t)}{S(q, 0)}=\exp \left[-\left(\frac{\Omega^{*}(q)}{a} t\right)^{0.85}\right] .
$$

For $\quad a \approx 1.354, \quad \Omega^{*}(q)=2 / \pi^{3 / 2} \Gamma(1 / 3)^{3 / 2} \Omega_{z} \quad$ with $\quad \Omega_{z}$ $=q^{3} k_{b} T(6 \pi \eta)^{-1}$, where $\Gamma$ is the Gamma function. This is the stretched exponential decay of the intermediate structure factor expected for Zimm dynamics in the $\Omega^{*}(q) t$ range relevant for NSE spectroscopy: $0 \leqslant \Omega^{*}(q) t \leqslant 6 .^{50}$ To allow a comparison to the theoretical predictions we also performed a first cumulant evaluation of the NSE spectra where the initial decay of the dynamic structure factor is fitted by a simple exponential decay. However, both the relaxation rates $\Omega^{*}(q)$ obtained by application of Eq. (22) as well as the relaxation rates $\Omega(q)$ obtained from a first cumulant evaluation show the same qualitative $q$ dependence. In Fig. 5, $\Omega(q)$, normalized to the $q^{3}$ dependence expected for internal Zimm dynamics, is plotted as a function of the unitless variable $q R_{g}$. As reference the normalized relaxation rates obtained for the homopolymer $g 0 n 144 f 1$ are also shown. The upturn at low $q$ values, corresponding to larger length scales, is due to the increasing sensitivity of the NSE experiment to the c.m. diffusion of the overall dendrimer. Here, the first cumulant should be proportional to $q^{2}$ as expected for free translational diffusion. At large $q$ values, on local length scales within the dendrimer the reduced relaxation rates reach a plateau indicating that the internal segmental motions of the dendrimers follow Zimm dynamics. The absolute relaxation rates for the dendrimer and the homopolymer are the same. On intermediate length scales of the order of the dendrimer dimension, i.e., $q R_{g} \approx 3$, the reduced relaxation rates exhibit a minimum. Analogous observations have been made by Richter and co-workers ${ }^{42-44}$ for high-functionality star polymers where the slowing down of the reduced relaxation rates on length scales of the arm dimension was attributed to collective breathing motions of the arms relative to each other. The architecture of the dendrimers compared to that of star polymers is more complex, and as a consequence the spectrum of normal modes contributing to structural relaxation is more complex, too.

To get further insight which motions contribute to the slowing down of $\Omega(q) / q^{3}$ in the case of the dendrimers we extended the Rouse-Zimm model of Cai and co-worker ${ }^{40,41}$ formulated for starburst dendrimers of special geometry $(f=3, m=2)$ to starlike dendrimers with arbitrary architecture. The solid line shows the result of a fit using Eqs. (18) and (19) without preaveraging the hydrodynamic interaction tensor. For the monomeric friction coefficient the fit yields $\zeta=(3.9 \pm 0.2) \times 10^{-13} \mathrm{~N} \mathrm{~s} \mathrm{~m}^{-1}$ and the reduced monomeric friction coefficient $\zeta_{R}=\zeta /(6 \pi \eta l)=(4.7 \pm 0.4) \times 10^{-2}$ as a measure of the relative strength of the hydrodynamic interactions can be calculated. The bond length $l=0.75 \mathrm{~nm}$ is determined by the SANS experiment and the viscosity of toluene is $\eta=0.59 \mathrm{mPa}$ s at $T=293 \mathrm{~K}$. The monomeric friction coefficient obtained here compares very well to the values found for other polymers with NSE spectroscopy. ${ }^{50-52}$ Having in mind that there is only one adjustable parameter, we obtain good qualitative agreement between experiment and theory. First of all we have to keep in mind that a preassumption for the derivation of Eqs. (18) and (19) is Gaussian statistics. As already discussed for the interpretation of the static results Gaussian statistic is violated, in particular, in the case of the dendrimers with shorter spacers between the branching points. The experimentally determined minimum is sharper with a much less gradual transition to internal Zimm dynamics than predicted by theory. However, the description of the depth of the minimum is reasonable. Richter and co-workers report a similar finding for high-functionality star polymers. ${ }^{42-44}$ For the star polymers both the sharpness as well as the depth of the minimum of the measured $\Omega(q) / q^{3}$ are underestimated compared to theoretical predictions. ${ }^{44}$ These deviations were attributed to an overestimation of hydrodynamic interactions between segments in the theory. High-functionality star polymers as well as dendrimers comprise high internal segment densities and as a consequence the backflow field of the solvent molecules is partly screened. Hydrodynamic interactions ease the renormalization of the reduced first cumulant by the form factor, ${ }^{53}$ leading to a less pronounced minimum than found experimentally. ${ }^{42-44} \mathrm{La} \mathrm{Ferla}^{27}$ predicted from his calculations within the Rouse-Zimm theory formulated for dendrimers with variable stiffness that the minimum in the reduced relaxation rate decreases in width and depth with increasing stiffness. Increasing stiffness prevents backfolding of the dendrons and, thus, reduces the internal segment density which in turn leads to stronger hydrodynamic interactions between the segments and a flattening of the minimum. However, according to the results deduced from the SANS experiments the dendrons on the dendrimers in particular, in those with shorter spacers, adopt a more stretched conformation compared to the high-functionality star polymer. Thus, hydrodynamic interactions leading to a flattening of the minimum in $\Omega(q) / q^{3}$ should be less screened which is not in accordance with our findings that the theoritical description of the depth of the experimentally determined minimum is reasonable.

In the following we would like to discuss the detailed 


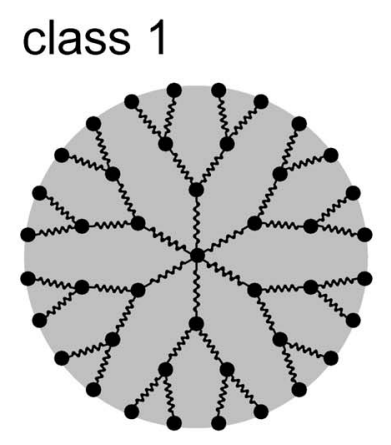

$Z_{0}=0$

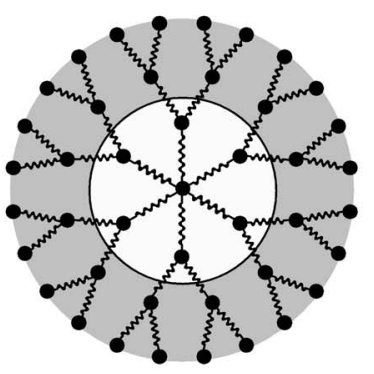

$Z_{0}=1$

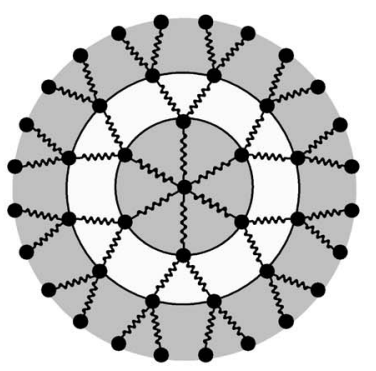

$Z_{0}=2$

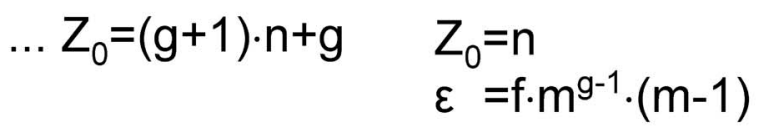

FIG. 6. Schematic sketch of the class 1 and class 2 eigenmodes.

nature of the normal modes contributing to the structural relaxation of the dendrimers. As sketched in Fig. 6 we can distinguish two classes of eigenmodes according to the behaviors of their collective displacements of the monomers. The first class comprises nondegenerate modes describing motions in which monomers belonging to different concentric subshells around the center bead are collectively moving together undergoing a sort of shearing motion. The subshells are not equivalent to the generation shells but are rather defined by the number of zeros $Z_{0}$ of the normal modes along a substrand built by $(g+1) n+g=N_{0}$ monomers in the starlike dendrimer. Thus the normal modes of class I comprise $N_{\text {class } 1}=N_{0}+1=(g+1)(n+1)$ modes including the mode with no zeros along a substrand. In the latter all monomers in the

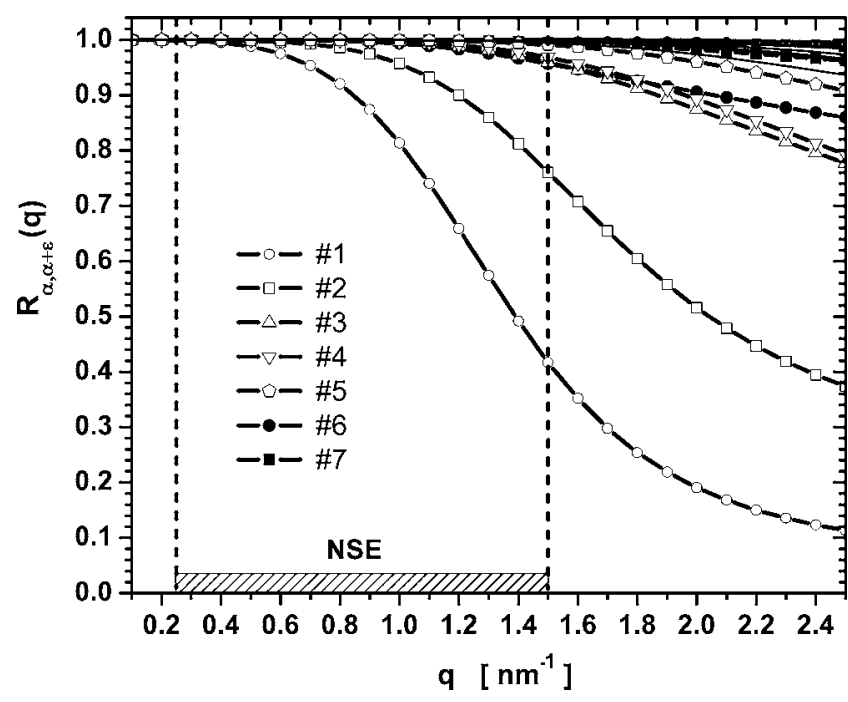

FIG. 7. Contribution to the relaxation of the intermediate structure factor of modes belonging to the same eigenvalue as a function of $q$ calculated using Eq. (20). The thick lines with empty symbols mark the class 2 eigenmodes: (a) core unit as stem with non zero \#1 and one zero \#3, (b) first generation branching unit as stem with non zero \#2 and one zero \#5, and (c) second generation branching unit as stem with non zero \#4. Higher order class 2 modes are displayed as thin solid lines. The solid lines with filled symbols mark the class 1 eigenmodes with one zero \#6 and two zeros \#7, etc. The $q$ window of the NSE experiment is marked by the shaded area.

dendrimer are moving in the same direction, thus, this mode describes the c.m. diffusion. The second class comprises degenerate eigenmodes describing motions where monomers belonging to subdendrons originating from the same stem (either core or branching unit) are collectively moving against each other in a sort of breathing motion. There are $N_{0}$ modes each with degeneracy $\varepsilon=(f-1)$ where the whole dendrons stemming from the central core are undergoing collective breathing motions. All other modes where the branching units build the stems comprise $N_{i}=(g+1-i) n+g-i$ modes each with degeneracy $\varepsilon=f m^{i-1}(m-1)$ where $i=1, \ldots, g$ is the generation of the group of branching units building the stems. Thus, the second class of eigenmodes comprises $N_{\text {class2 }}$ modes,

$$
\begin{aligned}
N_{\text {class 2 }}= & N_{0}(f-1) \\
& +\sum_{i=1}^{g}[(g+1-i) n+g-i] f m^{i-1}(m-1),
\end{aligned}
$$

and $N_{\text {class } 1}+N_{\text {class } 2}=N_{t}$ is the total number of normal modes contributing to the structural relaxation of the dendrimers.

We now would like to address the question which normal modes contribute to the decay of the intermediate structure factor in the $q$ range of the NSE spectrometer. In Fig. 7 the contributions $R_{\alpha, \alpha+\varepsilon}(q)$ of all modes belonging to the same eigenvalue $\lambda_{\alpha}$ of degeneracy $\varepsilon$ to the decay of $S(q, t)$ are plotted as a function of the scattering vector. The mode contributions $R_{\alpha, \alpha+\varepsilon}(q)$ were calculated using Eq. (20) which was derived assuming $\theta$ conditions. However, it can be seen from Fig. 1 of Ref. 38 that there is no difference in the ordering of the lower numbered modes between good and $\theta$ solvent conditions. The dynamics becomes faster on local length scales but slows down on larger length scales due to 
the expansion of the dendrimers by going from $\theta$ to good solvent conditions. In summary, even though the mode contributions in Fig. 7 are calculated under the assumption of $\theta$ conditions they should give a representative representation of which normal modes give a significant contribution to the relaxation dynamics of the dendrimers on length and time scales of the NSE experiment. Obviously, the breathing modes (class 2) give the main contribution due to their high degree of degeneracy. Here, only those modes are relevant where the dendrons originating from the center bead, and first and second branching units are moving relative to each other with no zero or only one zero along the substrands. The contribution of the shearing modes (class 1) is rather small.

The dynamic behavior found here for the starlike dendrimers is different to that found for starburst dendrimers. In a previous publication on starburst PAMAM dendrimers with generations between $g=5$ and 8 only c.m. diffusion was observed even though length scales larger and much smaller than the overall dendrimer dimension were probed. ${ }^{8}$ The absolute values obtained for the diffusion coefficients were equal to those expected for homogeneous, hard spheres. No internal dynamics were detected. An explanation can be found by looking at the differences in the dendrimer structures. In Fig. 8 the radial density distribution and the radial mass distribution are shown for the starlike dendrimer $g 2 n 10 f 6$ and the starburst PAMAM dendrimer $g 5 n 1 f 4$. To allow a comparison between the two dendrimers, distances from the center of mass are normalized to the radii of gyration. The mass distribution is normalized to the same mass. Compared to the starburst dendrimers the density in the starlike dendrimers decays much faster and a major fraction of the mass is located at much larger distances from the center in a region with reduced polymer concentration. For the starburst dendrimers the polymer concentrations in the rather extended core region become very high, almost meltlike, and the smoothly decaying surface region is rather small. As a consequence, the internal dynamics is too slow compared to the c.m. diffusion to be resolved by the experiment. In the starlike dendrimers the density is significantly reduced and the internal dynamics becomes comparable to and, for high $q$ values, faster than the c.m. diffusion.

Stark et $a l .{ }^{9}$ performed NSE experiments on carbosiloxane dendrimers with generations $g=1,2$, and 3 with perfluorinated end groups in perfluorohexane. Deviations from simple c.m. diffusion found for the $g=3$ dendrimer were attributed to form fluctuations and discussed in the framework of a model used to describe the shape fluctuations of microemulsion droplets. ${ }^{54,55}$ The contribution of the relaxations of ripples in the surface to the decay of $S(q, t)$ is only significant at $q$ values where the form factor has zeros or minima, at other $q$ values it is masked out by c.m. diffusion. Shape fluctuations give rise to a, compared to the c.m. diffusion, extrafast relaxation thus, the normalized relaxation rate $\Omega(q) / q^{2}$ exhibits a local maximum in the vicinity of the minima of the form factor. The $g=3$ carbosiloxane dendrimer exhibits a rather spherical shape and the form factor shows the characteristic minima. For the $g=1$ and 2 carbosiloxane dendrimers the form factors do not show any minima which was attributed to a more elliptical shape of the low genera-

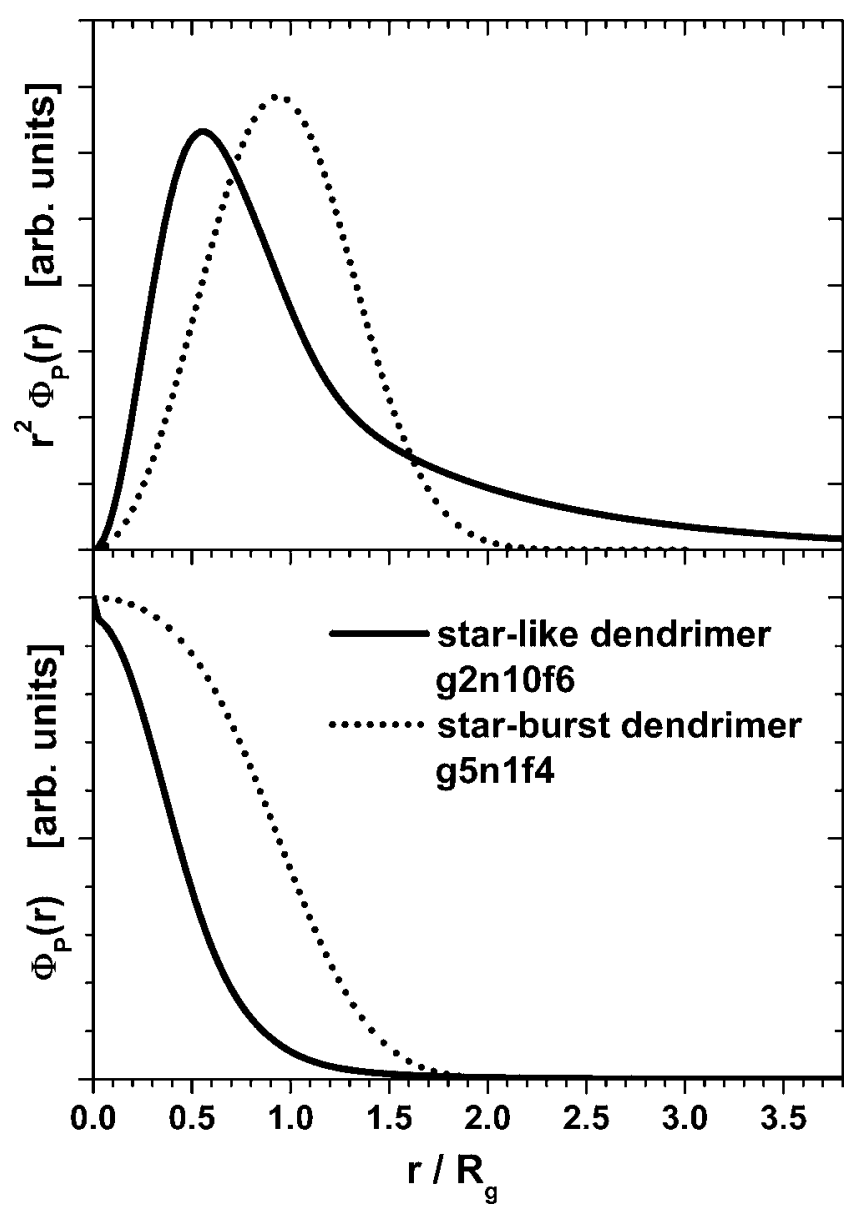

FIG. 8. Comparison of the radial mass distributions (top) and density profiles (bottom) obtained for a starburst PAMAM ( $g 5 n 1 f 4)$ and a starlike dendrimer $(g 2 n 10 f 6)$. Radial distances from the center-of-mass are normalized to the radii of gyration for each dendrimer. Taking into account the different architectures, the profiles are also normalized to the dendrimer masses.

tion dendrimers and the dynamic experiment reveals c.m. diffusion only. The starlike dendrimers investigated here have rather extended density profiles and consequently the form factors do not show any minima but exhibit the typical power-law decay expected for internal density fluctuations. They are mass fractals and not surface fractals and do not exhibit a sharp surface. So we do not expect that shape fluctuations are a relevant relaxation mechanism for the starlike dendrimers. In the $q$ range $q R_{g} \approx 3.6$, estimated from the hypothetical first minimum of the sphere form factor with $R$ $=\sqrt{5 / 3} R_{g}$, in which the shape fluctuations should give rise to an extrafast relaxation, we clearly observe a local minimum of the normalized relaxation rates due to the collective breathing modes of the dendrons. However, as mentioned above in a previous study on the internal dynamics of starburst PAMAM dendrimers we did not observe shape fluctuations either. Even though the PAMAM dendrimers have a rather globular shape and the form factors show well-defined minima, only c.m. diffusion was observed in the whole $q$ range of the NSE experiment. Stark et al. ${ }^{9}$ attributed their findings to the fact that the end groups in their particular dendrimers are coated by the end groups due to the unfavorable interaction of the end groups with the core, thus forming 
a sort of compact shell. However, the interpretation of their NSE data in terms of form fluctuations would imply not only a well-defined incompressible surface formed by the end groups but also a low viscosity liquidlike interior. Thus, it cannot be excluded that the extrafast relaxation process found by Stark et al. ${ }^{9}$ is due to internal density fluctuations and not to shape fluctuations.

\section{CONCLUSIONS}

We investigated the structure and internal dynamics of starlike poly( $\varepsilon$-caprolactone) dendrimers in dilute solutions with SANS and NSE spectroscopy. Toluene was chosen as good solvent. The SANS data were evaluated in the framework of the Beaucage model ${ }^{49}$ and by rigorous evaluation of the static form factor from its definition via the connectivity matrix which contains the information about the topology of the dendrimer. For the latter approach and for the interpretation of our NSE results we generalized the Rouse-Zimm model of Cai and Chen $^{41}$ for starburst dendrimers $(f=3, m$ $=2$ ) to starlike dendrimers with arbitrary architecture in terms of the following parameters: generation number $g$, spacer monomer number $n$, and functionalities of core and branching units, $f$ and $m$, respectively. The Rouse-Zimm approach gives direct access to the single normal modes contributing to the structural relaxation of the dendrimers and enables us to assign which relaxation mechanisms are relevant in the $q$ and time range of the NSE experiment.

The starlike dendrimers become more compact with increasing generation and increasing spacer length. On the other hand deviations from Gaussian behavior become larger with decreasing spacer length. The dendrons in the starlike dendrimers with short spacers stretch out to gain volume, therewith circumventing overcrowding. Dendrimers with longer spacers adopt a more space filling shape most likely by backfolding of the dendrons to the center. This results in a more Gaussian-type conformation of the dendrons in the dendrimers with long spacers. Increasing generation, accompanied with a gain in volume, does not lead to further stretching of the dendrons.

From a first cumulant evaluation of the NSE spectra for each scattering vector $q$, separately the relaxation rates $\Omega(q)$ can be obtained and, thus, the NSE experiment yields information about the dynamics of the dendrimers on different length scales $\propto q^{-1}$. The normalized relaxation rates $\Omega(q) / q^{3}$ exhibit a pronounced minimum on length scales corresponding to the dendrimer dimension. The Rouse-Zimm approach reveals that this slowing down of the dynamic relative to the internal Zimm dynamics is due to collective breathing modes where (parts of the) dendrons originating from the same stem are collectively moving against each other. Stems can be either the central core or the groups of branching units belonging to the same generation. Shape fluctuations are not a relevant relaxation mechanism for the starlike dendrimers which are mass fractals and exhibit a rather extended density profile.

\section{APPENDIX: CONSTRUCTION OF CONNECTIVITY MATRIX}

The monomers in the starlike dendrimer are numbered in the way sketched in Fig. 1. The core is numbered 1, subsequently the monomers in each linear spacer of the zeroth generation are numbered in a consecutive manner before numbering the branching units of the first generation and so on. The connectivity matrix $\boldsymbol{A}$ can be constructed in analogy to the procedure described in Ref. 41 for dendrimers with arbitrary generation number $g$, spacer monomer number $n$, and core and branching functionalities $f$ and $m$, respectively, in the following way. All unspecified matrix elements are equal to zero.

Diagonal elements:

- Core unit $A[1,1]=f$.

- Branching units for $g>0: A[i, i]=m+1$ with $i=N_{t}(j)+1, \ldots, N_{t}(j)+N_{\text {ter }}(j)$ and $j=0, \ldots, g-1$.

- Terminal units: $A[C(g)+i \cdot n, C(g)+i \cdot n]=1$ with $i=1, \ldots, N_{\text {ter }}(g)$ and $C(g)=N_{t}(g-1)+N_{\text {ter }}(g-1)$.

- Linear units in the terminal generation: $A[C(g)+j+i \cdot n, C(g)+j+i \cdot n]=2$ with $i=0, \ldots, N_{\text {ter }}(g)-1, j=1, \ldots, n-1$, and $C(g)=N_{t}(g-1)+N_{\text {ter }}(g-1)$.

- Linear units in the internal generations for $g>0$ :

$A[i, i]=2$

with $i=N_{t}(j-1)+N_{\text {ter }}(j-1)+1, \ldots, N_{t}(j)$ and $j=0, \ldots, g-1$.

Nondiagonal elements:

The connectivity matrix $\boldsymbol{A}$ is symmetric, thus, $A[i, j]=A[j, i]$.

- Connections to core unit: $A[1,2+i \cdot n]=-1$ with $i=0, \ldots, f-1$.

- Connections to branching units for $g>1$ : $A[C(k)+i, D(k)+1+(i \cdot m+j) \cdot n]=-1$, $A[C(k)+i, D(k-1)+(i+1) \cdot n]=-1$ with $k=0, \ldots, g-1, i=0, \ldots, N_{\text {ter }}(k)-1, j=0, \ldots, m-1$, $C(k)=N_{t}(k)+1$, and $D(k)=N_{t}(k)+N_{\text {ter }}(k)$.

- Connections between linear spacer units: $A[C(k)+i \cdot n+j, C(k)+i \cdot n+j+1]=-1$ with $k=0, \ldots, g, i=0, \ldots, N_{\text {ter }}(k)-1, j=1, \ldots, n-1$, and $C(k)=N_{t}(k-1)-N_{\text {ter }}(k-1)$.

Here $N_{t}, N_{\text {ter }}$, and $N_{\text {br }}$ are defined as follows:

$$
\begin{aligned}
& N_{t}(y)=\frac{f\left[\left(m^{y+1}-1\right) n+m^{y}-1\right]}{m-1}+1, \\
& N_{\text {ter }}(y)=f m^{y}, \\
& N_{\text {br }}(y)=f\left(m^{y}-1\right) /(m-1) .
\end{aligned}
$$

For $y=g, N_{t}$ yields the overall number of monomers constituting the dendrimer, $N_{\text {ter }}$ the number of terminal units, and $N_{\text {br }}$ the total number of branching units. 
${ }^{1}$ G. E. Buhleier, W. Wehner, and F. Vögtle, Synthesis 2, 155 (1978).

${ }^{2}$ G. R. Newkome, C. Moorefield, and F. Vögtle, Dendrimers and Dendrons (Wiley, New York, 2001)

${ }^{3}$ Dendrimers and Other Dendritic Polymers, in Wiley Series in Polymer Science, edited by J. M. Frechet and D. A. Tomalia (Wiley, West Sussex, 2001).

${ }^{4}$ T. J. Prosa, B. J. Bauer, E. J. Amis, D. A. Tomalia, and R. Scherrenberg, J. Polym. Sci., Part B: Polym. Phys. 35, 2913 (1997).

${ }^{5}$ T. J. Prosa, B. J. Bauer, and E. J. Amis, Macromolecules 34, 4897 (2001).

${ }^{6}$ D. Pötschke, M. Ballauff, P. Lindner, M. Fischer, and F. Vögtle, Macromolecules 32, 4079 (1999).

${ }^{7}$ S. Rosenfeldt, N. Dingenouts, M. Ballauff, N. Werner, F. Vögtle, and P. Lidner, Macromolecules 35, 8098 (2002).

${ }^{8}$ S. Rathgeber, M. Monkenbusch, M. Kreitschmann, V. Urban, and A. Brulet, J. Chem. Phys. 117, 4047 (2002).

${ }^{9}$ B. Stark, C. Lach, B. Farago, H. Frey, C. Schlenk, and B. Stühn, Colloid Polym. Sci. 281, 593 (2003).

${ }^{10}$ S. Rathgeber, T. Pakula, and V. Urban, J. Chem. Phys. 121, 3840 (2004), and references therein.

${ }^{11}$ R. Scherrenberg, B. Coussens, P. van Vliet, E. Guillaume, J. Brackman, and E. de Brabander, Macromolecules 31, 456 (1998).

${ }^{12}$ M. Ballauff and C. N. Likos, Angew. Chem., Int. Ed. 43, 2998 (2004), and references therein.

${ }^{13}$ R. L. Lescanec and M. Muthukumar, Macromolecules 23, 2280 (1990).

${ }^{14}$ M. L. Mansfield and L. I. Klushin, Macromolecules 26, 4262 (1993).

${ }^{15}$ M. L. Mansfield, Macromolecules 33, 8043 (2000).

${ }^{16}$ D. Boris and M. Rubinstein, Macromolecules 29, 7251 (1996).

${ }^{17}$ M. Murat and G. S. Grest, Macromolecules 29, 1278 (1996).

${ }^{18}$ K. Karatasos, D. B. Adolf, and G. R. Davies, J. Chem. Phys. 115, 5310 (2001).

${ }^{19}$ G. Giupponi and D. M. A. Buzza, Macromolecules 35, 9799 (2002).

${ }^{20}$ E. G. Timoshenko, Y. A. Kuznetsov, and R. Connolly, J. Chem. Phys. 117, 9050 (2002)

${ }^{21}$ A. V. Lyulin, G. R. Davies, and D. B. Adolf, Macromolecules 33, 6899 (2000).

${ }^{22}$ S. V. Lyulin, L. J. Evers, P. van der Schoot, A. A. Darinskii, A. V. Lyulin, and M. A. J. Michels, Macromolecules 37, 3049 (2004).

${ }^{23}$ P. K. Maiti, T. Çağin, S.-T. Lin, and W. A. Goddard III, Macromolecules 38, 979 (2005).

${ }^{24}$ W. Burchard, K. Kajiwara, and D. Nerger, J. Polym. Sci. Part B: Polym. Phys. 20, 157 (1982).

${ }^{25}$ P. G. De Gennes and H. L. Hervet, J. Phys. (Paris), Lett. 44, L351 (1983).

${ }^{26}$ B. Hammouda, J. Polym. Sci. 30, 1387 (1992).

${ }^{27}$ R. La Ferla, J. Chem. Phys. 106, 688 (1997).

${ }^{28}$ T. C. Zook and G. T. Pickett, Phys. Rev. Lett. 90, 015502 (2003).
${ }^{29}$ A. D. Meltzer, D. A. Tirrel, A. A. Jones, P. T. Inglefield, D. M. Hedstrand, and D. A. Tomalia, Macromolecules 25, 4541 (1992).

${ }^{30}$ A. D. Meltzer, D. A. Tirrel, A. A. Jones, and P. T. Inglefield, Macromolecules 25, 4549 (1992).

${ }^{31}$ C. B. Gorman, M. W. Hager, B. L. Parkhurst, and J. C. Smith, Macromolecules 31, 815 (1998).

${ }^{32}$ J. D. Epperson, L.-J. Ming, G. R. Baker, and G. R. Newkome, J. Am. Chem. Soc. 123, 8583 (2001).

${ }^{33}$ M. Wind, K. Saalwächter, U.-M. Wiesler, K. Müllen, and H. W. Spiess, Macromolecules 35, 10071 (2002).

${ }^{34}$ C. Malveau, W. E. Baille, X. X. Zhu, and W. T. Ford, J. Polym. Sci., Part B: Polym. Phys. 41, 2969 (2003).

${ }^{35}$ S. V. Lyulin, A. A. Darinski, A. V. Lyulin, and M. A. J. Michels, Macromolecules 37, 4676 (2004).

${ }^{36}$ M. A. Mazo, M. Yu. Shamaev, N. K. Balabaev, A. A. Darinskii, and I. M. Neelov, Phys. Chem. Chem. Phys. 6, 1285 (2004).

${ }^{37}$ M. Han, P. Chen, and X. Yang, Polymer 46, 3481 (2005).

${ }^{38}$ F. Ganazzoli, R. La Ferla, and G. Raffaini, Macromolecules 34, 4222 (2001).

${ }^{39}$ F. Ganazzoli, Condens. Matter Phys. 5, 37 (2002).

${ }^{40}$ Z. Y. Chen and C. Cai, Macromolecules 32, 5423 (1999).

${ }^{41}$ C. Cai and Z. Y. Chen, Macromolecules 30, 5104 (1997).

${ }^{42}$ D. Richter, B. Stühn, B. Ewen, and D. Nerger, Phys. Rev. Lett. 58, 2462 (1987).

${ }^{43}$ B. Ewen and D. Richter, Neutron Spin Echo Investigations on the Segmental Dynamics of Polymers in Melts, Networks and Solutions, in Advances in Polymer Science, Vol. 134 (Springer, Berlin, 1997).

${ }^{44}$ D. Richter, B. Farago, L. J. Fetters, J. S. Huang, and B. Ewen, Macromolecules 23, 1845 (1990).

${ }^{45}$ S. Rathgeber, A. P. Gast, and J. L. Hedrick, Appl. Phys. A: Mater. Sci. Process. 74, S396 (2002).

${ }^{46}$ M. Trollsås and J. L. Hedrick, J. Am. Chem. Soc. 120, 4644 (1998).

${ }^{47}$ J. S. Pedersen, Journal de Physique IV - Proceedings 3, 491 (1993).

${ }^{48}$ M. Monkenbusch, R. Schätzler, and D. Richter, Nucl. Instrum. Methods Phys. Res. A 399, 301 (1997).

${ }^{49}$ G. Beaucage, J. Appl. Crystallogr. 28, 717 (1995); 29, 134 (1996).

${ }^{50}$ D. Richter, M. Monkenbusch, A. Arbe, and J. Colmenero, Neutron Spin Echo in Polymer Systems, in Advances in Polymer Science, Vol. 174 (Springer, Berlin, 2005).

${ }^{51}$ D. Richter, B. Farago, R. Butera, L. J. Fetters, J. S. Huang, and B. Ewen, Macromolecules 26, 795 (1993).

${ }^{52}$ D. Richter, R. Butera, L. J. Fetters, J. S. Huang, B. Farago, and B. Ewen, Macromolecules 25, 6156 (1992).

${ }^{53}$ P. G. de Gennes, Physica (Amsterdam) 25, 825 (1959).

${ }^{54}$ S. A. Safran, J. Chem. Phys. 78, 2073 (1983).

${ }^{55}$ J. S. Huang, S. T. Milner, B. Farago, and D. Richter, Phys. Rev. Lett. 59, 2600 (1987). 